Rev. salud pública. 14 sup (1): 98-111, 2012

\title{
La formación por competencias y el mundo del trabajo: de la calificación a la empleabilidad
}

\author{
Competency-based training and work world: from grading to \\ employability
}

\author{
John H.Estrada M.
}

Departamento de Salud Colectiva. Facultad de Odontología. Universidad Nacional de Colombia. Bogotá. jhestradam@gmail.com.

Recibido 21 julio 2009/Enviado para Modificación 5 Mayo 2010/Aceptado 9 Diciembre 2010

\section{RESUMEN}

En cuanto elemento del discurso empresarial, la educación basada en las competencias surge asociada a procesos de reestructuración productiva que caracterizaron la economía a partir de la década de 1970, dada la crisis del modelo de acumulación basado en la producción y consumo en masa cimentados en los principios del taylorismo y del fordismo. En las décadas recientes al Estado le resulta imposible cumplir con las tareas de resolver las crisis económicas periódicas del capitalismo tardío lo cual hace que el Estado se aparte de su misión económica y patrocine los mecanismos de mercado, al mismo tiempo que trata de manejar la crisis de motivación poblacional. Este desafío lo obliga a interesarse en el mundo vital de los individuos para intentar resolver la crisis de legitimidad, mediante reformas educativas que afectan el mundo del trabajo. Queda explícita la relación entre los cambios vertiginosos del mundo laboral y una nueva formación educativa que considere al mismo tiempo la capacidad de gestión, de aprender, de trabajo grupal y de autocapacitarse. Se deriva una relación directa entre los cambios tecnológicos, la crisis estructural del capitalismo y la organización del trabajo y se sustituye el término de calificación por el de formación por competencias.

Palabras Clave: Formación por competencias, neoliberalismo, mundo del trabajo, taylorismo, fordismo, reforma, educación, empleo (fuente: DeCS, BIREME).

\section{ABSTRACT}

Considered as an element of business discourse, the competence-based education emerges associated with processes of productive restructuring influencing the economy since 1970. These processes arise as a consequence of the crisis of the accumulation model based on mass production and consumption following the principles of taylorism and fordism. In the last decades, the State has been unable 
to solve the periodic crisis that afflicts late capitalism. Because of this, the State moves away from its economic mission, promotes marketing mechanisms and, in the meantime, it tries to manage the motivational crisis of the population. This challenge forces the State to take interest in the vital world of individuals trying to solve the legitimacy crisis through educational reforms that affect the world of work. The relationship between the vertiginous changes of working world and a new educational formation is explicit. This educational formation must consider (at the same time) the management capacity, learning capacity, teamwork capacity and self-training. Based on this situation, there is a direct relationship between technologic advances, the structural crisis of capitalism and work organization. Besides, the "qualification" term is replaced with "competency-based education".

Key Words: Competency-Based Education, education, reform, employment (source: Mesh, NLM)

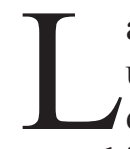

a competencia, tal como ha sido presentada en la literatura, designa un conocimiento inseparable de la acción, asociado a una habilidad, que depende de un saber práctico, o de una facultad más general, que el inglés designa con el término agency. De ese modo, se designan las capacidades para realizar una tarea con ayuda de herramientas materiales y/o instrumentos intelectuales. En ese sentido, la competencia es aquello por lo cual un individuo es útil en la organización productiva (1).

Desde la perspectiva de las pedagogías críticas, es importante introducir el debate sobre la formación de las competencias, pues subrayan sus principales exponentes, que detrás y en el fondo del debate sobre la formación de los jóvenes, se encuentra una teoría del sujeto, una teoría del conocimiento y una manera de relacionarlas. Pero claro, es también una teoría sobre el poder, ya que se asiste a una disposición estratégica del lenguaje en el campo institucional de la educación, para reforzar o articular determinados dispositivos de poder y control de los sujetos. La sociedad moderna está enmarcada por intereses e ideologías determinadas y se ha producido una penetración en la educación del término competencias, penetración que no resulta sorprendente dados los cambios vertiginosos en el modelo económico, en el mundo del trabajo y por supuesto en el mundo de la escuela (2). De qué se habla y de qué se deja de hablar, quién habla, desde dónde, en qué marco de relaciones históricas, estas son preguntas centrales al encarar el debate del enfoque por competencias a la luz de las pedagogías críticas (3).

A medida que la sociedad se torna más compleja y más integrada, el trabajo profesional se hace más diferenciado y más sujeto a cambios y las 
demandas sobre los nuevos profesionales cambian constantemente. Para el nuevo orden ya no basta con los conocimientos de expertos adquiridos en la educación inicial. Actualmente es necesario contar con las habilidades que permitan poner esos conocimientos al servicio de circunstancias no habituales, así que se requiere de flexibilidad, habilidades comunicativas y trabajo en equipo. También se requiere la capacidad de dejar de lado los conocimientos en algún momento de la vida y estar preparado para adquirir nuevas formas de experiencia y conocimiento y para desarrollar nuevas capacidades. En otras palabras, la reproducción del capital no requiere del conocimiento per se como en épocas anteriores, sino de las habilidades necesarias para sacarle provecho y si es necesario para dejarlo de lado. Esta es una sociedad de lo descartable, también en lo cognitivo (4).

En términos educativos se empieza a hablar de competencias en el año 1992, cuando una comisión del Ministerio del Trabajo de Estados Unidos (comisión SCANS - The Secretaries Commission on Achieving Necessary Skills-) determina un conjunto de destrezas que deben ser adquiridas tempranamente en el sistema educativo para hacer frente a las exigencias del cambiante mundo del trabajo. Según esta comisión, el mejoramiento de la calidad de la educación que apunte a la formación de competencias prácticas reduce la deserción escolar, genera individuos competentes en el mercado laboral, y como resultado, los productos y servicios brindados por Estados Unidos serán más competitivos a nivel mundial. Las metas productivas dependen del factor humano, de que los trabajadores se desempeñen cómodamente con la tecnología y los sistemas complejos de producción, siendo capaces de trabajar en equipo y con una sed insaciable de seguir aprendiendo (5).

Más adelante, en 1996, la comisión de educación para el siglo XXI de la Organización de las Naciones Unidas para la Educación, la Ciencia y la Cultura (UNESCO) publica el informe Delors, en el cual se considera a la educación como una herramienta necesaria para la materialización de las reformas, que será necesario implementar en el área de recursos humanos para que los trabajadores se preparen frente a la globalización (6).

Queda explícita la relación entre los cambios del mundo laboral y una nueva formación educativa que considere al mismo tiempo la capacidad de gestión, la capacidad de aprender y la capacidad de trabajo grupal; se deriva una relación directa entre los cambios tecnológicos y la organización del 
trabajo, y se sustituye el término de calificación por el de competencia. Para el Banco Mundial, un trabajador que quiera desempeñarse en la economía mundial y en la sociedad global debe dominar habilidades de índoles técnicas, interpersonales y metodológicas. Las habilidades técnicas comprenden las habilidades relacionadas con la alfabetización, idiomas extranjeros, matemáticas, ciencias, resolución de problemas y capacidad analítica.

Entre las habilidades interpersonales se cuentan el trabajo en equipo, el liderazgo y las habilidades de comunicación. Las habilidades metodológicas abarcan la capacidad de aprender por su propia cuenta, de asumir una práctica de aprendizaje permanente y de poder enfrentarse a los riesgos y al cambio (5).

En cuanto elemento del discurso empresarial, la formación basada en las competencias surge asociada a procesos de reestructuración productiva que caracterizaron la economía a partir de la década de 1970, dada la crisis del modelo de acumulación basado en la producción y consumo en masa cimentados en los principios del taylorismo y del fordismo. En las décadas recientes al Estado le resulta imposible resolver las crisis económicas periódicas del capitalismo tardío (7), esto lleva a una crisis de motivación en la sociedad y hace que el Estado se aparte de su misión económica y patrocine los mecanismos de mercado y trate de manejar la crisis de motivación interesándose en el mundo vital de los individuos para intentar resolverla. El modus operandi del Estado ha pasado de un interés distante respecto del valor económico, a un interés en el tipo de vida que promueve el proceso de educación, hasta tal punto que hoy interviene no sólo en el carácter de la experiencia educativa de los estudiantes, sino en la vida misma de estudiantes y docentes a través de profundas reformas a las instituciones educativas, a los currículos y a los proyectos educativos para hacerles funcionales al sistema de acumulación vigente (4).

El actual periodo de desarrollo industrial conocido como toyotista, (surge en Japón después de la segunda guerra mundial de la mano de su mentor, el ingeniero Taiichi Ohno) está caracterizado por un cambio general del sistema organizativo de la producción, que facilita la fragmentación del proceso y su difusión en pequeñas unidades independientes y coordinadas para la producción denominada "justo a tiempo", esto es sólo bajo demanda y evitando stocks voluminosos y controlando la calidad de los productos en el mismo proceso de producción (8). 
Igualmente se introduce el concepto de trabajo en pequeños grupos de trabajadores que deciden tareas múltiples y controlan varias máquinas y secuencias al mismo tiempo (9). Esta es la forma de organización de las grandes empresas transnacionales, así como la del sector productivo a escala nacional e internacional, cuyas principales características son: una desagregación de empresas en red, que actúa de manera descentralizada y basada en la subcontratación; la constitución de empresas por funciones empresariales; la concentración del poder financiero y administrativo en un núcleo reducido y distante a los eslabones productivos; desarrollo de redes de comunicación que integran los fragmentos productivos y las funciones dispersas; la producción en tiempo real que quiere responder de manera inmediata a la demanda; y, un debilitamiento de las garantías para los trabajadores de las empresas (10).

Las competencias hacen parte de un nuevo escenario social que pretende mediar las conflictivas relaciones entre el capital y el trabajo, este último desvinculado de la noción de empleos permanentes o, ligados a un puesto de trabajo, y que responda de manera permanente a las fluctuaciones de las demandas por nuevas capacidades operativas de los trabajadores (11). Basado en principios como flexibilidad e integración, el enfoque por competencias gana fuerza en escenarios de contracción del empleo, precarización de las relaciones laborales, desmovilización sindical y de los movimientos de izquierda en un momento de avance del discurso y retórica neoliberal (12). La flexibilización implica la constante adaptación de las tareas laborales a productos, procesos y mercados cambiantes, el incremento de la autonomía de los trabajadores, altos niveles de manejo de habilidades y capacidades de auto-programación y responsabilidad individual.

La globalización del comercio y de la industria, y la exigencia de un desarrollo productivo fundado en el conocimiento, ha generado en los actores económicos un interés por el desarrollo de las capacidades educativas de la sociedad, provocando demandas al sector educativo, en la perspectiva de adecuar su organización y funcionamiento en respuesta a los nuevos desafíos de la economía (13).

El capitalismo actual necesita refundar la escuela y su proyecto educativo, porque él ya cambió los fundamentos de su sociedad, de su cultura, de su proyecto de desarrollo y del ser humano, es decir, su proyecto ha sido transformado desde la ciencia y la tecnología. 
Los nuevos trabajadores deben ser formados en consideración a los nuevos sistemas de organización productiva y en relación con las demandas crecientes por conocimiento y manejo de tecnologías cada vez más sofisticadas en los lugares de trabajo (10). El concepto de competencia que se ha introducido en las propuestas educativas, remite a saberes, habilidades $\mathrm{y}$ actitudes individuales nuevos para el desempeño laboral y pretende hacer frente a los desafíos de competitividad contenidos en la nueva organización mundial del trabajo (14).

El capitalismo globalizado construye un nuevo patrón de acumulación y de control centrado en la tecnología, en el cual, cada vez más, se relega en el proceso a quienes no poseen las competencias propias de ese mundo tecnológico. Este capitalismo globalizado no garantiza el pleno empleo, mientras que el capitalismo manufacturero, a pesar del desempleo que tenía como ejército de reserva, funcionaba buscando el pleno empleo (15). Hoy el capitalismo tiene claro que la educación no garantiza empleo, y por ende, la escuela adquiere una función distinta. Es una escuela para la reestructuración productiva y los trabajadores deben garantizar el aumento de la productividad. Por eso, la educación de hoy es una educación para la empleabilidad, no hay más educación para el trabajo en el sentido tradicional (9). La empleabilidad es formar seres humanos con las capacidades, las competencias de saber hacer, saber técnico, saber de pericia y saber de cualidades comunicativas para salir a disputar los pocos puestos de trabajo que quedan.

Aparecen claramente tres tipos de competencias en las cuales se conjugan el proyecto globalizador y el proyecto de empleabilidad: las competencias cognitivas, las técnicas y las de gestión. Con estas competencias se constituye la idea de meritocracia, mediante la cual se avalan en el individuo los saberes que posee y se convierten en el pasaporte para ingresar a la educación superior o al mundo de la empleabilidad. Va a ser la posesión de las competencias lo que determine el tipo de méritos para ubicarse en los diferentes niveles y estamentos de la escala social.

Lo que queda en cuestión es la creencia de que alguna competencia puede habilitar a los jóvenes para avanzar en un mundo cambiante. El profundo cambio social, internacional y ecológico, invalida la noción misma de competencia, si se considera, que el término designa a algún conjunto de comportamientos, actividades o respuestas que tendrán mañana el mismo valor que hoy tienen (4). 
Como consecuencia del cambio de calificación a empleabilidad, las formas de trabajo simple comienzan a ser relegadas generándose nuevas formas de desempleo o de incorporación laboral; los trabajadores son sometidos a tipos de explotación de siglos anteriores: se paga por pieza terminada, no les son reconocidas las luchas por los derechos sociales ya ganadas, generando una precarización del mundo del trabajo y por lo tanto una reelaboración de la teoría del trabajo y una reestructuración de las clases sociales $(9,16)$.

El discurso de las competencias se presenta como un elemento de la nueva realidad capitalista que pretende responder a las exigencias de la realidad cambiante, y por lo tanto, busca establecer nuevas prácticas de formación; promover el progreso económico, elevar las capacidades humanas para el trabajo y la vida en general, lo cual según sus apologistas, contribuirá a la construcción de una sociedad más justa por la disminución de las desigualdades, el aumento de ingresos de la población trabajadora y por consiguiente, mayor equidad en la sociedad en su conjunto.

Tales promesas se configuran como elementos de un proceso de ideologización de la educación, constituido entre otras cosas por las ideas de la vinculación entre educación y desempeño económico y de la posibilidad de recalificación permanente de los trabajadores (12). Desde esta lectura economicista, competencia remite a la idea de progreso, elevación de la calificación de los recursos humanos, instrumento para lograr mayor productividad y competitividad de las empresas (10).

Por otra parte, este mismo discurso de las competencias se convierte en factor de atraso, marginalidad y exclusión del mundo del trabajo para un porcentaje significativo de personas, quienes no son capaces de adaptarse o adecuarse a las condiciones que ofrece este nuevo escenario. Uno de los efectos más significativos en el mundo del trabajo, es la reproducción de un importante segmento de trabajadores informales, junto con un contingente de trabajadores que se incorporan a empleos del sector formal en sectores rutinarios de producción y de servicios en una situación de precarización, bajos ingresos y desprotección social, y una minoría de empleados de alta calificación y buenos ingresos, quienes trabajan en identificación y resolución de problemas y cuyo mercado es global $(8,11,17)$.

Este cuadro estructural de exclusión condiciona el acceso restringido al empleo, particularmente a los jóvenes de escasos recursos y limita las 
posibilidades reales de incidencia de los esfuerzos educacionales por superar los problemas de desigualdad. Los jóvenes de América Latina (y Colombia no es la excepción) de las clases menos favorecidas, pese a contar con mayores posibilidades de acceso y permanencia en la educación formal o de acceso a capacitación y calificación laboral, se han visto expuestos a cambios que reducen drásticamente sus expectativas de obtener empleos estables y con cobertura de seguridad social mínima o nula (10).

La prolongación de la dependencia en los hogares de origen, el impulso de estrategias de sobrevivencia familiar, la integración precaria al mundo del trabajo con entradas y salidas esporádicas, la violencia barrial, las infecciones de transmisión sexual (incluido el VIH) y el embarazo adolescente son sólo algunas de las consecuencias de esta exclusión (18).

\section{LAS RACIONALIDADES QUE SUBYACEN AL ENFOQUE DE LAS COMPETENCIAS}

La lógica de la incorporación de las competencias en las políticas educativas ha sido conducida con evidentes marcas teóricas derivadas de tres corrientes que aportan características particulares, y que en ocasiones entran en disputa entre ellas: 1. El racionalismo, 2. El individualismo y; 3. El neopragmatismo (12).

\section{Del racionalismo}

De esta escuela de pensamiento se toma la idea de objetivación de las competencias, materializada en las tentativas de normalización de las competencias que explican los actos humanos, particularmente los del mundo del trabajo, en una secuencia lógica que permite la introducción de la eficacia por la vía del control y el autocontrol de las actividades en una tentativa de máxima racionalización de la producción y la formación.

Esto penetra en el discurso de la escuela porque el gobierno y la empresa se vieron necesitados de información objetiva que permitiera reconocer el nivel de calificación, escolaridad o dominio de contenidos de los egresados, más capacidades efectivas que facilitarán la planeación y diseños productivos en entornos más flexibles.

Esto se tradujo en la objetivación de propiedades antes difíciles de objetivar y de medir tales como las competencias relacionadas con el 
desempeño profesional. Este tipo de análisis parte del presupuesto de que las acciones y los comportamientos considerados competentes pueden ser objetivamente observados, evaluados, registrados, prescritos y enseñados.

Detrás de este discurso se puede evidenciar una racionalidad instrumental pues en la educación para la vida, el concepto de resultados objetivados tendría un espacio limitado o ningún espacio, dado que en la cotidianidad de los individuos, asistimos a procesos continuos, con puntos intermedios donde detenerse a revisar y modificar antes de continuar con el análisis y no a una sumatoria de pequeños procedimientos independientes y no relacionados (4). Esta descomposición de tareas se hace natural y se traduce en una didáctica que se basa en la organización modular y jerárquica de los procesos de aprendizaje en microprocedimientos intermedios que pueden al sumarse contribuir al logro de "ser capaz" de los alumnos (12). Esta racionalización se inspira claramente en los procesos que ocurren en las empresas para ajustar los atributos de los individuos a la organización del mundo del trabajo (19).

El descomponer los procesos como estrategia, para tornar observables y manipulables las competencias, no da cuenta ni garantiza que la complejidad de los actos de los alumnos pueda ser reproducida y bien comprendida. El abordaje racionalista de la competencia desconoce que esta es una construcción social, y como tal no puede desligarse del contexto histórico-cultural ni de las relaciones sociales, por esto cuando se produce un catálogo o lista de habilidades y capacidades, que serían requeridas por la dinámica de los procesos de trabajo, estos aparecen desligados de contextos sociales y culturales y adquieren una identidad autónoma como si pudieran existir sin los sujetos (20).

Una idea de racionalización también inspira las prácticas de evaluación que se proponen medir objetiva y rigurosamente las competencias de alumnos y trabajadores. Se busca utilizar prácticas selectivas, y que aparecen como neutras, en detrimento de juicios de carácter subjetivo a fin de verificar objetivamente el grado de aproximación entre un desempeño y las expectativas en cuanto a un comportamiento competente. Una evaluación de este tipo pretende ser científicamente neutra y pierde su sentido en beneficio del cumplimiento de instrucciones (21).

Al utilizar procedimientos jerarquizados y objetivos definidos por especialistas, la pedagogía de las competencias gana o se arropa con status de procedimiento científico y esto tiene efectos sobre el estilo cognitivo profesional 
de los maestros y estudiantes y promueve procesos adaptativos que aproximan las prácticas de docentes y alumnos a las demandas del sistema productivo.

\section{Del individualismo}

De esta corriente se puede decir que subyace al enfoque por competencias, toda vez que la noción de competencia utilizada supone individualización en la formación, la evaluación y los balances realizados. Una pedagogía individualista promueve un proceso formativo en el cual se valoriza el desempeño de capacidades individuales y no sociales, desvalorizando las capacidades motoras, intelectuales y los comportamientos comunes a todos los individuos involucrados en un proceso formativo. La pedagogía de las competencias coloca al alumno en el centro del sistema educativo e instaura un principio de cursos individualizados en lugar de una habilitación colectiva para todos los estudiantes. A pesar de ser colocadas como individuales, como ya se mostró, las competencias requieren una validación colectiva de parte de la organización escolar antes de ser implementadas. Los procesos formativos pasan de tener como centro los saberes a tener ahora como centro a los alumnos en busca de una obstinada individualización y en detrimento de los elementos colectivos. "La formación y la evaluación por competencias, fundamentadas en procesos de negociación individualizada, contribuyen para moldear una cierta concepción de sujeto y de autonomía, que aportan al desarrollo de capacidades adaptativas y disminuyen las prácticas de solidaridad como consecuencia del aumento de competición entre los individuos" (12).

Esta lógica individualizante no choca con el tipo de solidaridad promovida por el capitalismo a favor del trabajo productivo en equipo, momento en el que se revaloriza lo grupal y las habilidades intersubjetivas para resolver problemas en equipo. Esta lógica de lo individual empobrece el sentido de lo colectivo entre los estudiantes y futuros trabajadores, rompe la solidaridad y promueve una concepción unificada de las necesidades de las empresas y de los trabajadores, enmascarando los intereses contradictorios entre capital y trabajo. Esto trae como resultado que las personas busquen enfrentarse de manera individual a los problemas que encaran y a que acepten la lógica por ejemplo de salarios diferenciales en su futura vida laboral basados en la productividad, como resultado de propiedades y acciones individuales (22).

Esta influencia individualista es susceptible de una crítica ontológica, ya que en esta filosofía de lo individual hay una debilidad conceptual respecto del carácter del ser humano. Esta perspectiva presenta una visión empobrecida 
de las acciones humanas, según la cual los individuos se ven impulsados a desempeñarse mediante estándares externos. Esta concepción niega a los individuos su capacidad: ellos no son los autores de sus propias acciones, ni siquiera de sus pensamientos. Los seres humanos desde esta perspectiva son simples actores, que no pueden desempeñarse reflexivamente. Se niega el poder de la razón crítica autogenerada, que puede acabar con los estándares de las competencias (4).

Del neopragmatismo

De esta corriente se puede afirmar en primer lugar, que aparece como una categoría contradictoria con el racionalismo, ya que el discurso neopragmatista se aproxima más al irracionalismo al considerar la imposibilidad de un conocimiento verdadero acerca de la realidad, colocando en descrédito las teorías que se proponen objetivas y cuestionando el edificio construido por las ciencias cognitivas en torno a la idea de competencias objetivadas considerándolas apenas como representaciones o narrativas. Lo que sucede con el enfoque por competencias es que realiza una combinación de ideas del racionalismo y del pragmatismo. De este último hay un rescate del utilitarismo, del inmediatismo, de la adaptabilidad, de la búsqueda de producir aprendizajes útiles, aplicables y que permitan el ajuste de los individuos a realidades extremamente dinámicas y flexibles. La pedagogía por competencias tiene su procedimiento básico centrado en las actividades que es también el procedimiento básico del pragmatismo en educación que busca justamente promover procesos de adaptación de los individuos frente a situaciones cambiantes y que traducidas al mundo del trabajo asumen la forma de recalificaciones permanentes y reconversión profesional de los trabajadores por las alteraciones de los contextos productivos (23).

Para Dewey y Rorty, representantes del pragmatismo y del neopragmatismo, las prácticas pedagógicas no cuestionan el orden burgués establecido y deben partir de las realidades observadas, a las cuales deben ajustarse los estudiantes y trabajadores durante sus vidas, pero no para transformarlas sino para responder a las demandas inmediatas de cambio y adaptación de los individuos a los contextos productivos del mundo del trabajo como se ha señalado. El pragmatismo en educación trae como consecuencia que se centralice el aprendizaje en el alumno y se descuide la tarea y el compromiso del docente y esto va generando con el tiempo una disminución de la calidad de la educación y en vez de promover la democracia como prometía ("promover un aprendizaje 
abierto", "aprender a aprender"), profundiza las desigualdades al dificultar que las clases populares accedan a los saberes socialmente acumulados en cuanto instrumentos de intervención de la realidad (24).

Con base en lo anterior, se puede concluir que un modelo educativo centrado en las competencias, se vuelve restringido y cuestionable cuando se intenta desarrollar como respuesta únicamente a los desafíos de productividad y competitividad, obviando la situación de crisis cultural y los desafíos de construcción de un orden social que integre a los sujetos (10). Un currículo basado en competencias es canal de un modelo de desarrollo económico y social que tiende a substituir valores tales como la ciudadanía social, la igualdad y la solidaridad por la competitividad. A pesar de que el enfoque por competencias introduce las ideas de trabajo en equipo, la comunicación y la elaboración conjunta, tales dimensiones no son más que una construcción ideológica, una ficción que oculta la fragmentación, el individualismo, el fracaso y la pérdida del vínculo social para la gran mayoría de sujetos sometidos a las nuevas reglas del sistema productivo mundial. Los programas que se estructuran en términos de competencias constituyen una tentativa por reproducir a nivel del sistema educativo, la ideología que domina actualmente en el mundo económico (19).

Por esto, las competencias y los resultados no pueden servir como guía para la elaboración de políticas y proyectos educativos. La educación debe desarrollar capacidades críticas, entre las cuales se han de contar la evaluación de las competencias contemporáneas y el posible rechazo de las mismas de parte de los alumnos. Las competencias seguirán siendo comportamientos y capacidades para actuar de maneras deseadas y definidas por otros. La idea de una competencia que pueda o permita acceder a lo impredecible es en sí misma incoherente. Tal como sucede con las competencias, los resultados representan una forma de cierre del telón definitivo. A partir de ellos se predeterminan las características que deben llegar a tener los estudiantes. Ambos términos por tanto, son parte de un lenguaje lleno de prejuicios, imposiciones y estrecheces. Surgen de una forma particular de razonamiento, la razón instrumental y buscan extender el dominio que ejercen en la sociedad, a la educación y de ese modo tornar marginales otras formas de acción y de razón (4).

Una política educativa interesada realmente en el desarrollo de las competencias de los futuros trabajadores debería considerar el conjunto de las capacidades humanas que involucran el desarrollo de los sentidos, las diversas formas de comunicación simbólica, el ejercicio del pensamiento abstracto, 
solucionar problemas, posibilidad de actuar de forma innovadora y autónoma como condiciones para establecer relaciones de cooperación con otros individuos que permitan la construcción de una verdadera ciudadanía y democracia comprometidas con obtener una sociedad emancipada de los límites sociales que hoy restringen el proceso educativo y el desempeño humano (25). Una condición para la construcción de una educación cualitativamente nueva pasa por la utilización de métodos formativos que favorezcan una comprensión de la dialéctica de los fenómenos, de su interdependencia e historicidad, e implica el reconocimiento de las conexiones que los datos aislados presentan entre sí, confiriendo sentido a las actividades de los espacios de producción en un contexto social amplio (26).

El reto de una política educativa debe ser la emancipación del control ejercido por las condiciones materiales de reproducción y dominación del capital, superando por tanto las referencias racionalistas, individualistas, pragmatistas y utilitaristas que han caracterizado a la educación basada en las competencias. Si se acepta que los intereses del mundo corporativo y del ámbito académico pueden convivir unos con otros y con los requerimientos del mundo de la vida, esos intereses no se pueden expresar a través de un lenguaje de competencias y resultados objetivados. Se requiere entonces del lenguaje de la educación, de un vocabulario completamente nuevo, que no semeje el guión de una obra de teatro donde los personajes leen sus parlamentos, sino más bien de una conversación entre los personajes de una novela que se va armando creativamente a medida que avanza la acción

\section{REFERENCIAS}

1. Martínez J. La formación del profesorado y el discurso de las competencias. Revista interuniversitaria de formación del profesorado. 2004; 18 (3): 127-143.

2. Estrada J. Elementos de economía política de la reestructuración capitalista. En: Caycedo J, Estrada J (Comps). Marx vive. Siglo y medio de manifiesto comunista. ¿Superación, vigencia o reactualización? Primera edición. Santafé de Bogotá, Colombia: Editorial Universidad Nacional de Colombia; 1999. p. 179-203.

3. McLaren P. Pedagogía crítica y cultura depredadora. Políticas de oposición en la era posmoderna. Primera edición. Barcelona, España: Ediciones Paidós Ibérica, S.A.; 1997.

4. Barnett R. Los límites de la competencia. El conocimiento, la educación superior y la sociedad. Primera edición. Barcelona, España: Editorial Gedisa; 2001. p. 286.

5. Vega R. Las competencias educativas y el darwinismo pedagógico. Revista Debates. Junio de 2008 [Internet]. Disponible en: http://www.revistadebates.com.ar/opinion/lascompetencias-educativas-y-el-darwinismo-pedagogico.html Consultado: Agosto de 2009. 
6. Delors J, Mufti IA, Amagi I, Carneiro R, Chung F, Geremek B y otros. La educación encierra um tesoro. Informe a La UNESCO de La Comisión Internacional sobre la Educación para el Siglo XXI. Santillana - Ediciones UNESCO, 2008.

7. Duménil G, Lévy D. Crisis y salida de la crisis. Orden y desorden neoliberales. Primera edición. México D.F., México: Fondo de Cultura Económica Editorial; 2007. p.322.

8. Antunes R. ¿Adiós al trabajo? Ensayo sobre las metamorfosis y el rol central del mundo del trabajo. Primera edición. Santafé de Bogotá, Colombia: Editorial antídoto; 2000. p.174.

9. Mejía M. Implicaciones de la globalización en el ámbito social y educativo y gremial. Ponencia presentada en Santiago de Chile en enero de 2004 en el XX Congreso Internacional de educación católica. [Internet] Disponible en: http://www.revistadocencia.cl/ pdf/20100730164742.pdf Consultado: Agosto de 2009.

10. Sepúlveda L. El concepto de competencias laborales en educación. Notas para un ejercicio crítico. Revista digital UMBRAL 2000. 2002; 8 [Internet]. Disponible en: http://www. reduc.cl. Consultado: Agosto de 2009.

11. Antunes R. Los sentidos del trabajo. Ensayo sobre la afirmación y la negación del trabajo. Taller de estudios laborales. Primera edición. Buenos Aires, Argentina: Ediciones herramienta; 2005. p.248.

12. Marcos R. As referencias da pedagogía das competencias. Revista Perspectiva. 2004; 22 (02): 497-524.

13. Hirsch J. El capitalismo hoy: ¿global y sin clases? En: Vega R (Eds.) Marx y el Siglo XXI. Hacia un marxismo ecológico y crítico del progreso. Primera edición. Santafé de Bogotá, Colombia: Editorial Antropos; 1998. p. 111-117.

14. Niño LS. Evaluación para la Rendición de Cuentas o Evaluación Autónoma y Colectiva. En: Niño LS (Comp.). Políticas Educativas Evaluación y Metaevaluación. Primera edición. Santafé de Bogotá, Colombia: Editorial Nomos Impresores; 2007. p. 131-144.

15. Mondragón H. Los ciclos económicos en el capitalismo. Las crisis, ¿cuándo y por qué? Primera edición. Santafé de Bogotá, Colombia: Ediciones Aurora; 2009. p.145.

16. Estrada JH. Salud y clase social en el mundo de hoy. Revista Opciones Pedagógicas. 2007; 35, 36: 152-178.

17. Antunes R. El caracol y su concha. Ensayo sobre la nueva morfología del trabajo. Revista Sociología del Trabajo. 2007; 59: 131-142.

18. Estrada $\mathrm{JH}$. Evaluación de programas educativos para jóvenes, como herramientas de prevención de VIH/SIDA. Revista Gerencia y Políticas de Salud. 2009; 8 (16); 72-90.

19. Giroux H. La escuela y la lucha por la ciudadanía. Tercera edición. México D.F., México: Siglo XXI Editores S.A. de C.V.; 2003.

20. Álvarez J. La evaluación en la sociedad neoliberal: tendencia envolvente en todos los ámbitos para la rentabilidad y la eficacia. En: Niño LS (Comp.). Políticas Educativas Evaluación y Metaevaluación. Primera edición. Santafé de Bogotá, Colombia: Editorial Nomos Impresores; 2007. p. 13-36.

21. Álvarez J. Evaluar para conocer, examinar para excluir. Segunda edición. Madrid, España: Ediciones Morata; 2005.

22. Chomsky N. El beneficio es lo que cuenta. Neoliberalismo y orden global. Primera edición. Barcelona, España: Ediciones Crítica; 2001. p. 194.

23. Niño LS. El sujeto en la evaluación educativa en la sociedad globalizada. Revista Opciones Pedagógicas. 2006; 32, 33: 39-54.

24. Apple M. Escuelas democráticas. Madrid, España: Ediciones Morata; 2005.

25. McLaren P. Pedagogía crítica, educación latina y política de lucha de clase. Revista Opciones Pedagógicas. 2005; 31: 103-126.

26. Vega R. Un mundo incierto, un mundo para aprender y enseñar. Las transformaciones mundiales y su incidencia en la enseñanza de las Ciencias Sociales. Volumen 2. Santafé de Bogotá, Colombia: Editorial Nomos; 2007. p.645. 